\title{
Leistungsverlagerungen können zum Problem werden
}

\section{Wolfram Strüwe}

Leiter Gesundheitspolitik Helsana

\author{
Das Bundesamt für Gesundheit (BAG) beauftragte Polynomics und Helsana, zu ana- \\ lysieren, ob nach Einführung von SwissDRG auffällige Mengenentwicklungen im \\ stationären Bereich und Leistungsverlagerungen in andere Bereiche feststellbar \\ sind. Diese aus theoretischer Sicht zu erwartenden Konsequenzen sind bisher nicht \\ im befürchteten Ausmass eingetreten.
}

\begin{abstract}
Download: www.bag. admin.ch/dam/bag/de/ dokumente/e-f/evalberkuv/kvg-spitalf/2017leistungsmengen-undverlagerungenzusammenfassung.pdf. download.pdf/2017zusammenfassungverhalten-spitaelerleistungsmengen-undverlagerungen-d.pdf
\end{abstract}

Die Einführung des Fallpauschalensystems SwissDRG war von grossen Hoffnungen begleitet: mehr Transparenz im stationären Sektor, stärkerer Wettbewerb unter den Spitälern und gesteigerte Kosteneffizienz. Man war sich aber auch bewusst, dass Letzteres nicht automatisch eintreffen muss. Denn in einem solchen Pauschalsystem werden auch Fehlanreize wie eine Tendenz zur Mengenausweitung (angebotsinduzierte

\section{Les transferts de prestations peuvent devenir problématiques}

L'Office fédéral de la santé publique a demandé à Polynomics et Helsana de se pencher sur la constatation éventuelle d'une évolution frappante du volume des prestations dans le domaine stationnaire et des transferts de prestations dans d'autres domaines après I'introduction du système SwissDRG.

L'évolution et les transferts théoriquement attendus jusqu'en 2014 n'ont pas atteint les niveaux prévus. D'un point de vue global, on n'a pas observé $\mathrm{d}^{\prime}$ augmentation du volume pouvant être clairement imputable au système tarifaire.

Les premiers transferts stratégiques se sont dessinés dans le domaine hospitalier ambulatoire en amont et en aval, ainsi que dans les soins stationnaires. Selon les auteurs, les répercussions financières des transferts partiels dans le domaine ambulatoire se chiffrent jusqu'à présent à 10 millions de francs. Les transferts de prestations traduisent uniquement une "fragilisation» des forfaits par cas. Les prestations déjà remboursées par le biais des forfaits par cas sont indemnisées une nouvelle fois, qui plus est par le biais des systèmes tarifaires des autres domaines de prestations.

Si ce problème de doubles remboursements n'est pas résolu en temps utile, des désagréments peuvent survenir, puisque ces remboursements peuvent être interprétés, sur le plan juridique, comme une violation de l'obligation de la protection tarifaire. Dans le cadre réglementaire, il y a lieu d'éviter, autant que possible, une telle violation de la protection tarifaire de la LaMal.

Nachfrage) und ungerechtfertigte Verlagerungen in vor- und nachgelagerte Leistungsbereiche gesetzt. Nach fünf Jahren war es Zeit, eine erste Bilanz zu ziehen, fand das Bundesamt für Gesundheit (BAG) und beauftragte Polynomics und die Abteilung Gesundheitswissenschaften von Helsana mit einer wissenschaftlichen Studie*. Ziel war es, zu analysieren, ob auffällige Mengenentwicklungen bei Leistungen im stationären Bereich zu beobachten sind, die direkt auf die Einführung von SwissDRG zurückgeführt werden können. Es interessierte zudem, ob Verlagerungen von Leistungen aus dem und in den stationären Bereich festgestellt werden können.

Die Anreize zur Mengenausweitung sind folgendermassen zu erklären:

- Im Fallpauschalensystem werden die variablen Kosten und die Fixkosten zusammen über die Fallpauschale pro Patientenfall vergütet. Unter solchen Systemvoraussetzungen liefert jeder zusätzliche Patientenfall, der einen positiven Deckungsbeitrag erwirtschaftet, einen Beitrag zur Finanzierung der (fallunabhängigen) Fixkosten.

- Das Fallpauschalensystem vergütet jeden Patientenfall mit einer fixen Pauschale, die unabhängig von den tatsächlichen, spitalindividuellen Kosten ist. Dies verursacht dem Spital finanzielle Risiken, die es vermindern möchte. Eine Strategie kann dabei sein, das Risiko über möglichst viele Patientenfälle zu poolen. Nach dem Gesetz der grossen Zahl können dadurch Verluste durch sehr teure Einzelfälle besser abgefedert werden.

Anreize zur Leistungsverlagerung wiederum bestehen aus folgenden Gründen:

- Im Fallpauschalensystem wird jeder Fall unabhängig von seinen tatsächlichen Kosten vergütet. Dies 
bietet den Spitälern die Möglichkeit, den Deckungsbeitrag pro Fall durch Kostenreduktionen zu steigern. Dies können sie einerseits - wie mit der neuen Spitalfinanzierung beabsichtigt - durch Effizienzsteigerung erwirken, sie können anderseits aber auch abgrenzbare Teilleistungen wie die Diagnostik, Laboruntersuchungen oder die Pflege an voroder nachgelagerte Leistungsbereiche auslagern.

- Die Spitäler haben zusätzliche Anreize zur Leistungsverlagerung, wenn durch eine Teilverlagerung das Behandlungsrisiko für das Spital reduziert und damit die Planungssicherheit erhöht werden kann. Dies gilt beispielsweise dann, wenn durch diagnostische Leistungskomponenten im spitalambulanten Bereich vor dem stationären Aufenthalt mehr über den Patientenfall und über allfällig zu erwartende Komplikationen in Erfahrung gebracht werden kann.

Als Datengrundlage der Wirkungsanalyse von Polynomics und Helsana dienten die medizinische Statistik der Krankenhäuser, die Krankenhausstatistik, die Fallkostenstatistik sowie diverse Statistiken zur Bevölkerungsstruktur und Abrechnungsdaten von Helsana aus den Jahren 2011 bis 2014.

\section{Leistungsverlagerungen und Mengenent- wicklung noch nicht besorgniserregend ...}

Die Ergebnisse erstaunen: Die aus theoretischer Sicht $\mathrm{zu}$ erwartenden Mengenentwicklungen und Leistungsverlagerungen bis 2014 sind (noch) nicht im erwarteten Umfang eingetroffen. Bei der Mengenentwicklung konnten noch keine klaren Tendenzen festgestellt werden. Zwar gibt es gewisse Spitäler, die stärker von Mengenausweitungen betroffen sind. Es ist jedoch unklar, ob diese auf das Tarifsystem zurückzuführen sind. Denn auf Gesamtebene zeigten sich keine Mengenausweitungen, die eindeutig auf das Tarifsystem zurückgeführt werden können. Der Betrachtungszeitraum ist noch zu kurz - so die Autoren -, um klare Effekte empirisch bestimmen zu können. Sie vermuten jedoch, dass sie mittelfristig auch in der Schweiz eintreten werden, wie dies die internationale Literatur für andere Länder mit stationären Fallpauschalen festhält.

Bei der Leistungsverlagerung zeichneten sich erste strategische Verschiebungen in den vor- und nachgelagerten spitalambulanten Bereich sowie in die stationäre Pflege $\mathrm{ab}$ :

- Mehr Patienten weisen seit SwissDRG vor- und/ oder nachgelagerte Leistungen auf.
- Von den Teilverlagerungen sind besonders Spitäler in Kantonen betroffen, in denen die stationären Leistungen vor SwissDRG mit Tagespauschalen vergütet wurden und welche damit nach Einführung von SwissDRG mit diametral gegensätzlichen Anreizstrukturen konfrontiert sind.

- Der spitalambulante Bereich ist von Teilverlagerungen besonders stark betroffen beziehungsweise wird für Optimierungen genutzt.

- Deutlich erhöht hat sich die Anzahl Patienten, die nach dem Spitalaufenthalt Pflegeleistungen im Pflegeheim beziehen, eine Erkenntnis, die sich mit anderen Studien und Auswertungen deckt.

- Bei den Gesamtverlagerungen fanden die Autoren keine Anhaltspunkte für Verlagerungen in den ambulanten Bereich, sondern eher Evidenz für Verlagerungen in den stationären Bereich, wobei die Effekte erheblicher Heterogenität zwischen den medizinischen Bereichen unterlagen.

Gesamthaft betrachtet sind die festgestellten Verlagerungen für das Gesamtsystem noch unbedeutend. Die finanziellen Auswirkungen der beobachtbaren Teilverlagerungen in den ambulanten Bereich veranschlagen die Autoren auf bisher rund 10 Millionen Franken. Sie empfehlen, die Analyse zu einem späteren Zeitpunkt zu wiederholen.

\section{... doch ein Problem zeichnet sich ab}

Obwohl es wohl noch Zeit braucht, bis sich die Wirkungen der neuen Spitalfinanzierung voll entfaltet haben, sind die Leistungsverlagerungen noch am sichtbarsten. Solche Verlagerungen sind aber problematisch, da sie unter den momentanen Bedingungen die Kosten der obligatorischen Krankenpflegeversicherung (OKP) in die Höhe treiben und über kurz oder lang ein Problem für die Spitäler werden.

\section{Gesamthaft betrachtet sind die festgestellten Verlagerungen für das Gesamtsystem noch unbedeutend.}

Die Verlagerungen von Leistungen in andere Bereiche, auch wenn sie nur schleichend erfolgen, bedeuten nichts anderes, als dass eine "Aushöhlung» der Fallpauschalen stattfindet. Leistungen, die bereits über die Fallpauschalen vergütet wurden, werden noch einmal entschädigt, und zwar über die Tarifsysteme der anderen Leistungsbereiche. Dies ist nichts anderes als eine Doppelvergütung, die nicht gerechtfertigt ist. 
Das DRG-System kann solche Verlagerungen nur mit einer zeitlichen Verzögerung auffangen. Grundlage der Systemkalkulation sind die stationären Kostenträgerrechnungen der Spitäler. Eine Leistung, die ehemals stationär erbracht wurde und daher zu Recht in die Kalkulation der Kostengewichte eingeflossen ist, dann aber ambulant erbracht wird, ist aus Systemsicht erst nach drei Jahren aus dem Kostenträger herausgerechnet. So lange dauert es, bis Veränderungen im System abgebildet sind.

\section{Der Lösungsansatz: Abschlag auf die Baserates}

Die Vergütung der Fallpauschale ist also für drei Jahre zu hoch, so dass die Spitäler während dieser Zeit einen Zusatzertrag erwirtschaften. Dies ist eine ungerechtfertigte Belastung der Prämienzahlenden. Da sich die Fallpauschale aus zwei Elementen zusammensetzt, nämlich den Kostengewichten aus der Tarifstruktur und der Baserate, gibt es prinzipiell auch zwei Ansatzpunkte zur Vermeidung des unerwünschten Effekts:

1. Es gäbe die Option, die Kalkulationsdaten aus den Kostenträgerrechnungen schneller in die Systemkalkulation einfliessen zu lassen. Aber dieser Lösungsversuch ist illusorisch: Der zeitliche Aufwand dafür ist zu gross. Die Daten der Spitäler müssten viel früher vorliegen, wie heute revidiert und dann der SwissDRG AG übermittelt werden. Sie sind dann gegebenenfalls nach Rücksprache mit den Spitälern zu plausibilisieren. Aber jede neue Systemversion muss auch eine Weiterentwicklung beinhalten, nicht nur bezüglich der medizinischen Entwicklung, sondern auch bei der Kalkulationsmethode und der ökonomischen Relevanz.
Bleibt also die Ebene der durch die Tarifpartner jährlich verhandelten Preise der Fallpauschalen, die Baserates. Differenzierte Baserates haben sich seit Einführung der neuen Spitalfinanzierung bei Problemlösungen bewährt: Unzulänglichkeiten - beispielsweise bestimmte Kostenunterschiede betreffend OKP-Pflichtleistungen -, die noch nicht auf Strukturebene gelöst werden konnten, können durch differenzierte Preise aufgefangen werden. Die Lösung ist also ein Abschlag auf die Baserate, der sich auf Leistungsverlagerungen in andere Bereiche zurückführen lässt. Wie hoch der Abschlag genau zu veranschlagen und wie er zu berechnen ist, bleibt Sache der Tarifpartner.

Wird das Problem der Doppelvergütung nicht rechtzeitig angegangen, kann Ungemach drohen: Die beschriebenen Doppelvergütungen sind rechtlich betrachtet als eine Verletzung des Tarifschutzgebotes interpre-

Leistungen, die bereits über die Fallpauschalen vergütet wurden, werden noch einmal entschädigt.

tierbar. In Artikel 44 Absatz 1 KVG heisst es nämlich: «Die Leistungserbringer müssen sich an die vertraglich oder behördlich festgelegten Tarife und Preise halten und dürfen für Leistungen nach diesem Gesetz keine weitergehenden Vergütungen berechnen (Tarifschutz).» Doppelvergütungen fallen unter den zweiten Halbsatz, auch wenn sie im Einzelfall schwer nachweisbar sein mögen. Eine Verletzung des Tarifschutzgebots des KVG sollte im heutigen regulatorischen Umfeld tunlichst vermieden werden. 